\title{
Key closure planning considerations
}

\author{
G.M. Bentel BHP Billiton, Australia
}

\begin{abstract}
Mining operations generally have long operating lives, and closure planning must be carried out to a sufficient level of detail to provide a reasonable level of accuracy of the costs of rehabilitation and closure.

In 2004 BHP Billiton mandated closure planning at all assets in compliance with a risk-based closure standard. In 2009 BHP Billiton embedded closure planning into its business systems by integrating closure engineering and planning into its life of asset planning process.
\end{abstract}

To ensure that all aspects of closure are fully considered in the planning process, BHP Billiton has developed a set of key closure planning considerations. This paper discusses these closure planning considerations and the importance of an integrated life of mine planning process.

\section{Introduction}

\subsection{Mine closure}

Mine closure is a process that starts when a mine decides to permanently terminate mining operations. At this point in time, the mine owners have a legal and social obligation to return their site to a safe, stable and self sustaining condition consistent with a post-closure landform and use agreed with the relevant regulatory authorities and other stakeholders.

The extent of the closure execution works required to achieve this end condition depends largely on the complexity of the closure issues and how well these have been defined, planned for and managed during the mine's operational life. The residual closure execution works depend on how much disturbance has been caused, how much progressive rehabilitation has been completed, how many closure issues remain, and how much certainty there is around successfully closing out these issues in a reasonable time and at a reasonable cost.

Once the closure execution works have been completed, the closure process continues into a care and maintenance period. The complexity of the closure issues determine the care and maintenance duration, as this is the time required to demonstrate that the completion or performance criteria have and will continue to be met in the very long term. The care and maintenance period can last for many years, and in some cases may extend in perpetuity.

Once the designated authority has agreed that the completion targets have been achieved, relinquishment of the mining tenement and other associated leases may occur. At this stage, liability for the tenement can be transferred to the original lease grantor, the original owner or a new owner, i.e. responsibility for the site can be transferred to the next land owner or user.

Closure planning is the whole-of-life process that progresses a closure plan through its various stages, from the definition of the closure fundamentals through to relinquishment. To minimise the risk associated with closure and at the same time optimise the cost of closure, the planning process should start during the project conception phases and continue throughout the lifecycle of the mining operation.

\subsection{Closure in BHP Billiton - the road to integrated planning}

In 2004, BHP Billiton mandated compliance with a risk-based closure standard (BHP Billiton, 2004) with a target group-wide compliance date set for the end of the 2007 financial year. The 2004 standard was aligned with all BHP Billiton group-wide policies and standards current at the time, and set objectives around the mitigation and management of current and future health, safety, environment and community (HSEC) and other business risks associated with closure. The standard also mandated the risk, valuation and project 
management processes to be followed in developing and maintaining closure plans at each asset or mining operation.

During 2009 the group level requirements for closure that were found in the 2004 closure standard were integrated into group level documents (GLDs) owned by the three principal and parallel corporate functions responsible for mine/asset closure:

- The Health, Safety, Environment, Community and Sustainability (HSEC\&S) function is responsible for closure management across the group. The HSEC\&S function facilitates the implementation of a consistent management approach to water, biodiversity and land management issues and to closure and HSEC legacy issues.

- BHP Billiton's Business Optimisation function is responsible for integrating closure planning into life of asset planning, i.e. for progressing the closure strategy and requirements developed by the HSEC\&S function, into an engineered plan.

- Group Reporting is responsible for calculating and reporting the closure and rehabilitation accounting provision in the annual financial statement.

The detailed closure development and planning steps discussed in Section 2 illustrate that within the broader process, the full range of group functions are consulted or participate directly in the development of the closure model. These functions include legal, project management, investment and value management, community, public relations, etc.

A fundamental lesson learned during the evolution of closure planning in BHP Billiton is that the key to a successful outcome, i.e. all closure activities are fully considered and appropriately planned, actioned, managed and funded, lies in the HSEC\&S, planning and accounting functions being aligned with a closure process fully integrated in the life of asset/mine planning.

\subsection{The business case for improved closure planning}

The operational life of mines "can extend for many years, typically from 5-20 years and in some cases more than 50 years" (Mine Closure Guideline, 2006). Closure execution works can take some years to complete, while the post-closure relinquishment process for more complex sites can last up to 10-15 years or more.

Unless the closure works are very simple and regulatory closure completion approval is virtually guaranteed, long range cost forecasting based on conceptual engineering and minimal planning and stakeholder engagement is likely to be significantly underestimated. The cost estimate will be based on assumed material types and quantities, unit rates estimated from conceptual scopes of works and assumed construction methodologies and schedules. The closure plan could therefore significantly undervalue the cost of closure if it does not adequately or accurately factor in all closure execution and post-closure care and maintenance activities.

More detailed closure planning may also end up being totally off target if the operation does not operate according to a plan that minimises closure impacts along the way. The late emergence of additional closure issues that need to be resolved can lead to significant increases in the scope and cost of closure execution works. When the issue grows to an extent where acceptable completion criteria cannot be agreed, the operation may be required to provide care and maintenance in perpetuity.

Research data (Mine Closure Guideline, 2006) "shows that almost $70 \%$ of mines that have closed in Australia over the past 25 years have had unexpected and unplanned closures". The sudden or early closure of a site caused by economic or other influences can dramatically increase closure costs through unplanned scope changes and associated costs not normally included in a closure plan. Such unplanned costs include redundancies, supply contract termination costs, and unrealised opportunities.

A large unplanned closure liability arising from the early closure of an operation can conceivably impact the viability of a smaller mining company, or alternatively it can materially impact the assessed portfolio value of a larger mining group. A large increase in the rehabilitation provision for a closed site can impact shareholders' dividend, perception of a company's profitability, and reputation. 
Therefore closure planning, which provides the framework for correctly estimating and valuing the financial implications of closure, is a key driver of any company's ability to provide for the closure of any mining operation. Closure planning also demonstrates appropriate governance of closure provisioning as required by external regulatory bodies, e.g. the US Securities and Exchange Commission (SEC).

\subsection{NPV - a disincentive to detailed closure planning?}

Planning for closure is really about planning and operating a mine to facilitate and enable successful closure. In the early stages of project conceptualisation, there is a lot of scope to enable simpler, more successful closure. For example, considering the cost of hauling materials to cover a tailing storage facility when deciding its position, or designing and positioning support infrastructure in relation to the post-closure community's beneficial needs. There are often instances where simple changes upfront can have profound implications for eventual site closure (ICMM, 2008).

Yet few operations take advantage of such opportunities, largely because closure value is normally measured by the net present value (NPV) of future cash flows discounted to present value. Discounting over very long periods (30-50 years) reduces large numeric values dramatically, so that large changes in the closure execution and post-closure costs have minor impacts on the estimated NPV closure cost.

As a result, there is understandably little incentive for long-life operations with many short-term production priorities to utilise skilled resources and budget on longer term closure planning, preferring to defer more detailed work until closer to closure.

While some view NPV as a barrier to the development of more detailed plans at an earlier stage, this deferral strategy is a logical and acceptable business approach, as long as it is well thought out and sensibly managed. The closure planning and decision making governance needs to ensure that:

- More detailed and accurate closure planning and provisioning is only deferred where appropriate.

- There are no increased risks or lost opportunities associated with deferring studies and more detailed closure planning.

- There is a high degree of confidence that deferral of more detailed closure planning will not lead to a material increase in closure execution, impact mitigation, or post-closure care and maintenance costs.

- The correct level of planning detail can be provided when required for making strategic investment decisions - either asset viability or expandability.

Irrespective of the deferral strategy, the closure plan must still evolve as mining progresses, so that the detail and accuracy is commensurate with the decision making requirements as the discount period shortens. Those assets within the portfolio that will be closing in the nearer term (less than 10 years) or that may be at risk of closing early because of changes in the profitability of the operation must be prioritised. For these assets, planning must ensure that all the necessary studies, engineering, planning and valuation can be completed in the remaining time to closure, to facilitate successful closure execution and relinquishment.

And while it may be strategically acceptable to defer more detailed closure planning for longer term operations, it is neither morally, nor legally acceptable, nor financially prudent, to defer the prevention or mitigation of any health, safety, environment, community or social closure impact. As soon as the potential for harm or unacceptable impact is observed or identified, appropriate preventative and/or mitigating controls must be implemented to minimise potential current and future harm. In other words, we must do whatever we can, as soon as we can, to avoid, prevent and mitigate the potentially harmfully effects of mining before they happen.

The reason for this is obvious - the current generation of mining companies are paying an enormous price to mitigate the legacy damage caused by the poor mining practices and inadequate closure planning and provisioning by earlier generations. We must not repeat this mistake and we must not knowingly leave a legacy of harm for future generations to deal with. 


\section{$2 \quad$ Key closure planning considerations}

To ensure that closure planning at each mine adequately covers the full spectrum of potential closure issues, BHP Billiton has developed a checklist of 28 key closure planning considerations. These are outlined below, under five principle closure planning drivers:

- Closure planning fundamentals.

- Risk management.

- Engineering, execution and project management.

- Cost estimate.

- Management.

As each key consideration is in itself a process requiring specialist input, the discussion does not pretend to provide this expertise, but rather provides context of its importance in the overall closure planning context.

\subsection{Closure planning fundamentals}

For the closure model to comprehensively cover all potential issues, the closure planning fundamentals underpinning the closure process and planning need to be fully defined and understood.

\section{Background/baseline conditions}

This describes and provides a database of the baseline pre-mining, or pre-operation, health, safety, environment, community and social conditions and historical data. The information and data must cover the areas that will be disturbed during the life of mine operational activities, including regional areas that may be impacted by the operation, e.g. groundwater, or by supporting infrastructure, e.g. port and rail. The background conditions provide the framework and basis for establishing and defining the minimum rehabilitation and closure requirements and performance criteria.

It is important to establish clear data requirements, data quality assessment standards and data management protocols focussing on parameters that are relevant to the valued HSEC systems specific to each operation.

Carefully planned data collection and monitoring will avoid delays in approvals/permitting by ensuring critical baseline data is available when needed. On the other hand extraneous data, or data not actively or attentively managed, uses up valuable time and effort when "outliers" need to be explained, or when low value data must be re-collected.

\section{Regulatory/legislative operating and closure framework}

This sets out the regulations, the legislation and the organisational requirements, policies, standards, guidelines, etc., that provide the legal and governance frameworks under which closure planning, execution and relinquishment will be carried out.

The earlier this framework is established, the more confidence there will be that the plan will fulfil the requirements, and the less risk there will be of a legal challenge to the closure plan at a later date.

\section{Life of mine disturbance and tenure}

Tenement details and plan(s) showing the licensed mining and supporting infrastructure locations, boundaries, tenement lease conditions, etc., both for current and proposed future life of mine disturbance areas that will be decommissioned, rehabilitated and closed.

Distinguishing between the current and future disturbance is important as closure provision is based on current disturbance only, while the life of mine planning must also account for future disturbance. 


\section{Closure commitments and obligations register}

This register of all legal and regulatory closure obligations, tenement conditions, commitments made in environmental impact assessments, and regulatory approval conditions, etc., is critical in contextualising the broad closure framework.

It is also very important to clearly document "constructive obligations" arising from compliance with an organisation's own standards that exceed minimum regulatory/legal requirements, as these demonstrate an organisations commitment to zero harm aspirations. It is also important to note any additional commitments and/or modifications arising from changes to the operation, and to explain the reasons behind these and the impacts that they have on the closure requirements.

As much detail as possible behind each commitment should be provided, so that when the closure plan is finalised sometime in the future, it is easy for others to understand the basis of the commitments and their intent. Unqualified or loosely worded commitments may lead to legal interpretations different from what was originally intended. For example, "similar" may be interpreted as "identical", leading to very different closure requirements and costs.

\section{Stakeholder analysis and engagement plan}

The objective of closure planning is to return the land to a form and use agreed with regulators and stakeholders. Stakeholders concerns, needs and aspirations must be taken into account when defining the landform, land use, and closure completion criteria, as later changes are likely to have material impacts on the scope, timing and cost of closure.

An adequate level of confidence in a closure plan and cost can only be achieved when measurable stakeholder engagement has occurred and the fundamental closure strategies and risks have been discussed. A framework for ongoing engagement and consultation also needs to be agreed.

The stakeholder engagement plan describes the roles and accountabilities of the team responsible for stakeholder engagement, and the action schedule that will ensure full engagement and agreement prior to feasibility level investment approval being sought for capital approval for closure execution.

Considerations 6 and 7 (objectives and criteria) require input from stakeholder engagement for their development, followed by ongoing consultation to ensure that they remain current and applicable.

\section{Closure objectives, strategy, land uses and landforms}

This is a statement and description of the overall closure objectives and rehabilitation and closure strategies for the agreed post-closure land uses and landforms (what successful closure looks like), as well as for individual disturbance domains within the operation that may have different landforms, uses and performance criteria.

It includes organisational objectives - charter, policy, standards, etc. - as well as particular objectives for the specific site developed through the consideration of closure, including progressive rehabilitation targets during the operational period. It is a commitment to all stakeholders of an agreed end position.

\section{Design and completion criteria}

Design criteria must be based on accepted standards, where possible avoiding numeric values if the accepted standard is likely to change with time. The design and completion criteria:

- Provide the basis for closure designs and method statements.

- Describe metrics against which the successful fulfilment of commitments, obligations and stated closure objectives will be assessed.

- Provide the basis for the post-closure monitoring and care and maintenance programs, and hence for the approvals required prior to relinquishment. 
Design and completion criteria should be listed for the whole site, e.g. the design storm for rehabilitation covers, the probable maximum flood, the maximum radiation level, permissible dust levels, etc., and as applicable to specific structures or features, e.g. the design life of a tailings dam cover.

It is recommended that valued health, safety, environment, community and social components, issues of importance, e.g. surface water, specific to the site are established early in the process. This helps maintain regulator and stakeholder focus on the key issues, reducing the risk of frequent changes to the desired end state. This also assists in focusing pathway analysis and ecological/radiological risk assessments onto key issues.

Self-imposed completion criteria that exceed the minimum regulatory, legal and internal organisational requirements, e.g. the requirement for a tailings storage cover to be suitable for pastoral use in outback Australia, should not be documented in this listing. These should be included where they apply to the evaluation of the cost/benefit of the alternative design or plan, so that they remain in context against other viable alternatives, e.g. offset pastoral land elsewhere on the lease, and do not automatically become interpreted as the minimum criteria at a later stage.

\subsection{Risk management}

The outcome of successful closure is that all health, safety, environment, community, social and business risk issues have been identified and managed in such a way that no measurable harm will be observed, or unexpected expenses incurred, in the many years after closure.

The key to successful closure risk management lies in minimising potential impacts before they occur or as soon as they are observed during the operational life. This includes planning studies, or research projects, to reduce the uncertainty and increase the knowledge around the various material closure issues.

\section{Lessons learned}

Past closure projects provide the precedent for both successful and unsuccessful closure strategies, methodologies and designs. A great deal of time, effort and value can be saved by benchmarking against, and learning from the experiences of past closure projects.

Corporate knowledge is easily lost in large organisations and individual mines are unlikely to have the required internal expertise and knowledge. Therefore it is important to establish long-standing working relationships with key consultants, thereby maintaining continuity of the closure history and tapping into a strong supporting experience and knowledge base.

Conversely it is unwise to develop in-house closure plans using personnel with inadequate knowledge, expertise and experience. These will only need to be reworked at a later stage if only to provide assurance to stakeholders that the design has been carried out by a reputable specialist.

\section{Closure risk management}

Closure risk management involves identifying and managing several categories/sets of closure risks, each of which require different treatments/controls and each of which has an impact on different closure planning cost aspects as noted:

- Operational risks that impact closure - the costs to treat and/or mitigate these affect the mine's operational budget.

- Closure design and planning risks - these affect the project execution quantities, specifications, schedules and unit rates.

- Closure execution project risks - these influence the closure execution project management contingency amount.

- Post-closure risks - these influence the post-closure risk provisional sum (an allowance for postclosure risk events that may/may not occur, e.g. repair damage to cover caused by erosion after 1-in-100 year storm). 
- Closure management risks - these affect the closure management cost contingency amount independent of closure execution project management costs.

It is important to correctly describe and focus on the key risk issues that have the potential to materially impact successful closure planning, execution and performance.

Risks are often confused with "causes", "pathways" and "impacts", and are often incorrectly stated as a risk when in fact simply a cost or quantity impact. For example, a shortage of material is not really a risk. If the design using the specific material has to be implemented, i.e. there is no alternative, then the material must be sourced from somewhere, although this may be at a higher cost than the insufficient quantity of waste material readily available on site.

When correctly stated/described, the number of risks to be treated and managed can be reduced to a manageable number - of the order of 5-10 key risks across whole of closure - encompassing the numerous minor risk issues within their controls. The design of risk treatments/controls for fewer well defined risk issues promotes the consideration of alternatives and/or opportunities and smarter design.

As mentioned before, the closure plan is in itself a risk management plan, and if the treatment of all risks within each domain or aspect is clearly explained, a separate risk management plan should not be required. In other words, a more powerful story can be told by describing exactly how a risk is going to be managed, rather than by trying to demonstrate this through complex rankings in a separate risk register.

The most important aspect of the risk management plan is to clearly schedule the closure risk control actions and accountabilities in the life of mine plan and shorter term plans, e.g. five year plan, and budgets. Accountabilities and key performance indicators set around completion of risk actions will ensure that they are completed as planned.

\section{Opportunities}

Many potential opportunities are available to reduce closure uncertainty, risk and cost. Examples include:

- Disposal/sale/gifting of beneficial infrastructure to the local government or community.

- Opportunistic revenue from the treatment of low grade ore, or the re-treatment of tailings - offsetting the cost of in-pit disposal.

- Alternative landform or land use - may require negotiation against commitments.

- Modification of operating methodology to reduce closure disturbance and hence rehabilitation requirements.

- Research and development of alternative technology to reduce uncertainty around closure methodologies.

- Smarter design - through the consideration of alternative closure strategies.

The importance of identifying and actioning opportunities that may materially reduce closure uncertainty, risk and cost as early as possible is clearly evident. Many opportunities, such as the full consideration of alternatives followed by agreement of a selected go-forward solution, require a fair amount of stakeholder engagement and study/research in order to provide assurance that they are legitimate, scientifically and technically sound, and acceptable to all parties.

\section{Remediation of current HSEC issues}

As discussed in previous sections, it is important to identify any current HSEC issues, e.g. ground water contamination, that may increase the closure risk, uncertainty and cost, and to mitigate and remediate these as soon as possible.

While the mitigation and remediation of current issues is an operational responsibility and activity, it is important to describe the proposed actions, completion criteria and requirements, and to track and document the process, outcomes and post-remediation monitoring for future reference. 


\subsection{Engineering, execution planning and project management}

The level of engineering, execution planning, and project management applied to the plan must be commensurate with the complexity of the closure issues and with the time remaining until closure execution (See discussion on NPV - Section 1.4).

Closure planning includes rehabilitation carried out during operation - progressive rehabilitation. It is prudent to invest in simple, effective and robust closure solutions that can be tested and optimised during operation for site-wide implementation at closure execution.

\section{Progressive rehabilitation}

Many organisations set progressive rehabilitation targets to return areas of disturbance to a state compliant with agreed post-closure landform and use as soon as they become available for rehabilitation. This is in line with regulatory and community desire to minimise the land disturbance at any given point in time.

The design of progressive rehabilitation must be able to achieve closure, i.e. to meet the closure criteria and performance requirements without being re-worked. Hence the design and construction must be carried out in a controlled fashion to enable accurate closure cost estimation, and to record the exact materials used so that monitoring can demonstrate success or failure.

The progressive rehabilitation must be integrated into the life of mine and short-term plans, e.g. five year plan. The planners must carry out annual reconciliation of actual rehabilitation versus forecast, and explain and justify any changes to the rehabilitation plan. This is important, as failure to carry out progressive rehabilitation during operations can have material impacts on the closure execution plan and cost.

\section{Closure execution methodology}

For each area/domain, zone or element that will be decommissioned, rehabilitated and closed, a step-by-step description is given of how the closure work will be carried out, and how the stated objectives and completion criteria will be achieved.

Each step normally has a corresponding line item - description, quantity and unit cost - in the cost model, as well as a specification for quality control and quality assurance purposed.

This is often the most comprehensive section of the plan, as it tells the story of how and when each area will be closed, the construction methodology, the materials to be used, what successful closure will look like, and how the risks will be managed, both during construction, and in the post-closure period.

\section{Design and engineering}

During the earlier stages of closure planning, concept level designs and material specifications need to be developed for each aspect requiring engineering. The level of engineering must be sufficient to provide a high level of confidence that the performance and completion criteria will be achieved. During the operational life, the design needs to progress, using data and performance information gathered from studies and full scale trials, from concept level engineering, through feasibility level, to detailed design prior to execution planning and cost estimation.

New technologies should be applied judiciously as unproven technology/methodology can lead to failure and significantly increased costs to re-do the closure work.

Smart engineering should aim to simplify maintenance requirements as complex engineering and control cannot be supported by a skeleton care and maintenance team. Smart design will also use natural features and topography (gravity) to the greatest advantage in the closure design to avoid ongoing energy costs and maintenance for pumping and other transfer systems.

Surface water management plays a large role in closure planning, and smart design will minimise the erosion potential by retaining surface water upstream of the rehabilitated areas (upstream retention is your best friend). 


\section{Studies}

Each mine generally has unique issues around its closure requirements and criteria. Hence while designs and methodologies developed elsewhere can be used to formulate a concept level design at another site, studies or research projects, are normally required at each site to:

- Provide specific data required for closure criteria, closure design and planning.

- Test any new alternatives or new technology proposed.

- Prove up concept level engineering using the local setting and available materials.

- Develop predictive models, e.g. groundwater required to defend closure strategies.

The objective of the studies is to reduce uncertainty in the proposed methodologies, engineering designs, estimated physicals and post-closure risk issues. This needs to be done prior to approval being obtained for closure execution capital, and hence the studies, some of which need to run for lengthy durations, need to be scheduled, resourced and budgeted within the mine's operating plans leading up to closure.

\section{Project management}

A description of the project management team - roles and responsibilities - and anticipated contract strategy - including execution manning - for supervising and carrying out the closure execution works.

This is important as the project management costs for an execution project running for some years can make up a significant proportion of the closure execution cost.

If an assumption is made that the closure works will be carried out using the residual operational staff and mining fleet and equipment, the risk of this not eventuating, i.e. closure execution requires external project management personnel and contractors, needs to be carefully assessed and managed, as the difference in cost can be material.

\subsection{Cost estimates}

Cost estimates factor in all closure activities and consider the potential range of influences on the closure execution works, as well as the post-closure costs associated with care and maintenance.

There are five primary outputs from the cost estimates:

1. Operational budget required to develop the closure plan, to manage current risks, to conduct ongoing closure management activities, e.g. legal, stakeholder engagement, studies, trials, etc., to perform provisional rehabilitation, and to manage the closure process.

2. Closure execution project costs including feasibility level design costs, reviews, approvals, project management costs and the closure execution project contingency amount.

3. The post-closure costs including monitoring, management, relinquishment planning and post-closure care and maintenance.

4. The life of mine closure cost comprising all costs associated with planning, progressive rehabilitation, closure execution, and post-closure care and maintenance.

5. The accounting rehabilitation and closure provision which appears as a liability in a company's annual report and is calculated as the cost to rehabilitate and close the current disturbance only calculated under international financial reporting standards (IFRS).

The accounting rehabilitation and closure provision is reviewed, and where required re-calculated annually; hence a robust closure plan and cost valuation model will simplify this process, and will provide proof of rigour and governance (auditability) required for annual financial reporting.

\section{Unit cost database}

The closure cost valuation model comprises numerous work scope items corresponding with the step-by-step closure methodologies and specification described in earlier sections. Each work item has a unit cost or rate 
derived from existing operating costs, industry benchmark rates, contractor quotations, or built up from first principles - using hourly equipment and labour rates, etc.

As the basis for unit costs can get lost in a large valuation model, it is recommended that a separate unit cost database be maintained, so that each unit rate/cost can be clearly described, and changes and updates clearly documented. The ranges of the costsm, see consideration 18 , used in the valuation model can also be defined and explained in the unit cost database.

In order to clearly differentiate the primary cost output categories - cost, risk provision and contingency unit costs must not include indirects - contractor inductions, etc. - project management costs, owner's costs, or contingency amounts. These must be calculated and itemised separately.

The unit cost database should be independently validated to ensure it is up to date and aligned with current operational and industry costs and rates. Unit costs developed by external sources can be difficult to update and simple updates using guessed across-the-board escalation factors can lead to significantly incorrect closure cost estimates.

\section{Range analysis and expected value}

The closure plan attempts to predict the cost of closure a long time into the future, using numerous assumptions, estimated rates, durations and quantities, all of which can be impacted by many influencing factors such as commodity price, exchange rates, weather, resource availability, etc.

Single point deterministic estimates inherently contain a large degree of uncertainty and financial risk is managed by catering for the range of possible outcomes for each variable, whether this is a unit rate, a physical quantity, a period for completion or a scheduled start/finish date. All BHP Billiton assets are required to use probabilistic modelling for closure provisioning calculations.

Range analysis estimates the average outcome by probabilistically weighting all the possible outcomes by their associated probabilities. In Figure 1, the expected value (mean) of Variable $\mathrm{X}$ is the average of the weighted probabilities of all possible outcomes of Variable X. Note that the range analysis also tests the extremes - the low probability outcomes that may conceivably occur.

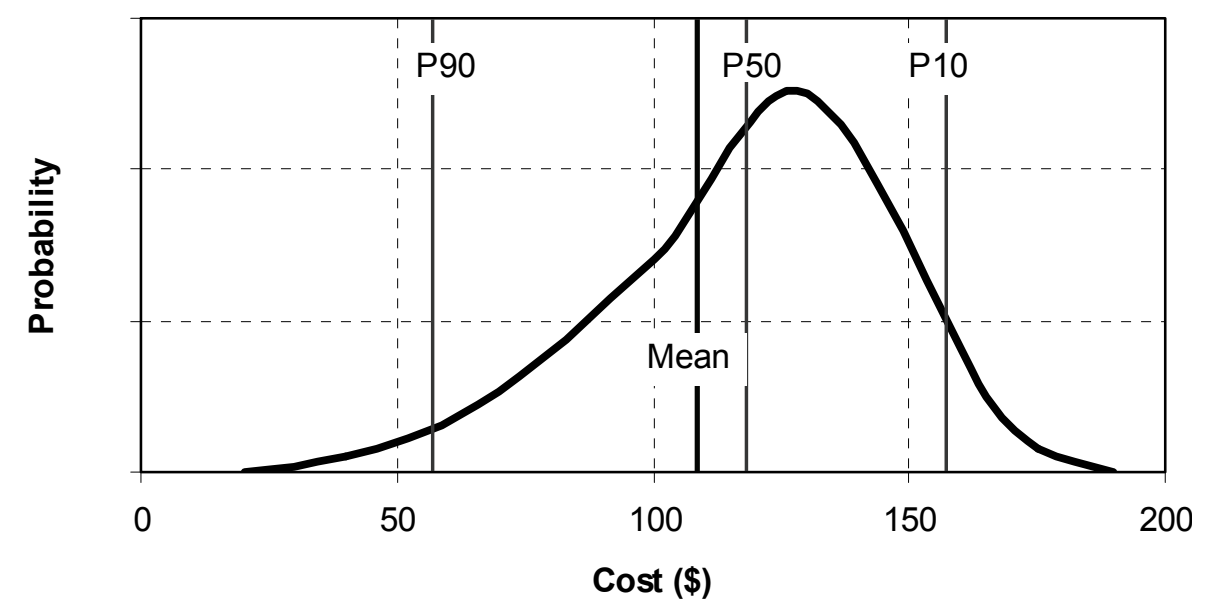

Figure 1 Probability distribution of Variable $X$

A thorough and robust range analysis not only allows the calculation of the expected life of asset closure cost, but also facilitates determination of the expected values of the individual principal cost items, i.e. progressive rehabilitation, closure execution, execution contingency, post-closure costs and closure provision.

\section{Closure valuation model}

The closure valuation model is a detailed cost valuation model that calculates the life of mine expected net present closure value. Using the expected values of input variables in each line item, and estimating the post- 
closure risk cost using probability weighted values for post-closure event risks, the model outputs a probability distribution of closure NPV, from which the expected - mean or weighted average - NPV can be obtained. In the normal distribution in Figure 2, the mean is the same as P50, but this does not hold for skewed distributions.

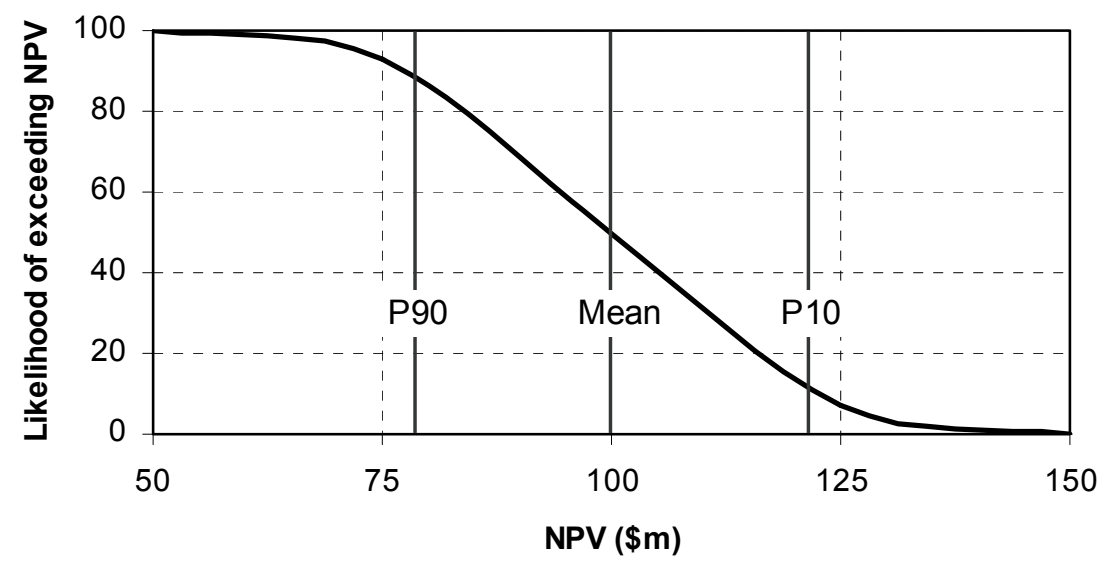

\section{Figure 2 Cumulative distribution of closure NPV}

Put simply, the expected NPV is the entire range of NPV outcomes weighted for the likelihood of each NPV outcome occurring. Thus, it incorporates the probability of the range of potential outcomes and their consequences.

In addition to the life of mine cost, other outputs from valuation model include expected NPVs for:

- Progressive rehabilitation.

- Closure execution project cost.

- Post-closure costs.

- Post-closure event risk provision.

- Closure accounting provision.

The above approach is very useful in decision making - with the closure cost presented this way management can make strategic decisions with respect to the closure risk profile that they wish to adopt. For example, while the mean or expected value is used in life of asset planning, consideration of when to close an operation nearing depletion of its ore reserves might use the P10 value to check a worst case scenario.

\section{Accounting provision}

The accounting restoration and rehabilitation provision is derived from the life of mine closure valuation, but using only the costs associated with restoring and rehabilitating the current disturbance. The provision appears in the annual financial reports as a liability.

While the closure provision calculation is a very important, the closure cost valuation model must not be set up primarily for provision calculation, as this may cause issues - such as contract terminations - that are not permitted to be included in the accounting provision balance, to be omitted from the life of mine model.

The closure cost valuation model must be set up for the life of mine case including all closure considerations, with the capability of switching off all line items not applicable to the provision calculation.

The provision excludes opportunities such as the re-treatment of tailings, any scrap value derived from the sale of scrapped plant and tax. The provision includes progressive rehabilitation, and is calculated using current costs - uninflated real costs - discounted from the anticipated closure date back to the present at a real discount rate which also does not include inflation.

The provision is adjusted annually to take into account actual inflation and any adjustments arising from rehabilitation completed or additional area disturbed. This means that if the disturbance area remains 
constant, the annual adjustments would cause the closure provision to increase as the duration to closure reduces, i.e. shorter discount period, and at closure, the provision would align with the life of mine cost adjusted to exclude those items not permissible in the provision, e.g. tax.

\subsection{Closure management}

Closure planning needs to be done by the right people working within the right team. Hence a documented long term management strategy for managing all aspects of the closure process will significantly reduce closure costs by reducing complexity and re-work, and by ensuring that all pre-closure activities are carried out in time to facilitate closure execution as soon as practicable.

Much value is wasted on studies whose duration is lengthened by incorrectly scheduled regulatory approvals, while attempting to fast-track closure studies is likely to end in failure to either gain approvals or to achieve agreed closure criteria. Value is also wasted on numerous restarts and reworking of the same data and information by successive teams, while lost data and information requires unnecessary and costly duplication.

The key to value maximising the closure planning process lies in fully integrating closure into the life of mine planning process, and in tracking key performance indicators that ensure that closure is considered within the planning and decision making framework at all times.

\section{Management team}

The roles and responsibilities of multi-functional management team set up to develop, review, update, improve and integrate closure planning towards readiness for closure execution should be described using the considerations outlined in this paper.

The team is likely to include representatives and contributions from HSEC, technical services, community, finance, projects, risk, supply, contracts, legal and management.

It is also necessary to define the manpower and skills required during closure execution and post-closure leading up to and including relinquishment.

\section{Post-closure monitoring plan}

The post-closure monitoring plan defines what needs to be monitored to confirm that the completion criteria and the performance requirements of closure elements are being achieved. This will logically align closely with the closure design and completion criteria, and hence developing the post-closure monitoring requirements and methodology early in the closure planning cycle will assist in focussing attention on key valued systems.

The plan defines the monitoring frequency, methodology, quality assurance and quality control standards, budget, data and analysis reporting requirements. In order to provide data continuity, the monitoring program needs to continue from the operational monitoring, through the execution works project, until relinquishment is achieved.

\section{Post-closure care and maintenance plan}

This plan describes the activities required to maintain the desired state of closure after completion of the execution works. It includes the anticipated duration - until relinquishment - scope, budget, and estimated frequency of care and maintenance activities, e.g. re-seeding, repairs to covers, or maintenance of drainage channels, etc.

The plan defines roles and responsibilities, maintenance frequencies, resources - personnel, materials and consumables - and the budget covering all these activities for input into the closure cost valuation model.

The care and maintenance plan must also cater for post-closure risk events - the infrequent impacts from major storms or earthquakes that exceed the design events during the post-closure period. The cost provision for these events is included in the valuation model as a risk provision, where the estimated cost of the repair is weighted by the probability of the event occurring during the post-closure maintenance period. 


\section{Relinquishment plan}

To avoid any unexpected liabilities/costs, closure planning needs to continue past closure execution, all the way to tenement relinquishment.

While the monitoring and care and maintenance plans take care of post-closure site related activities, it is also important to define the expertise and resources, e.g. legal, required to represent the operation in demonstrating compliance with completion, obtaining regulatory agreement and sign-off, and achieving final transfer of liability to the original or subsequent owner.

\section{Joint venture partnerships}

The responsibilities and obligations of each joint venture partner must be clearly nominated in the closure plan. There are many reasons that may contribute to a joint venture partner's need to delay or default on contributing to closure costs, and hence the possibility of this occurring should be treated as a risk within the closure plan and cost valuation model. To this end, the roles and responsibilities around liaison between/with joint venture partners should also be clearly defined.

\section{Community consultation plan}

Community consultation and negotiation around the potential impacts of closure on communities requires specialist skills as well as the authority to negotiate sensitive issues on behalf of the operation. This includes exploring and possibly committing to opportunities to reduce social and community impacts through support programmes, training and community participation.

Hence it is important to define the roles, responsibilities and strategy and plan for community engagement so that all personnel associated with closure planning are able to direct queries on social/community issues through the correct channels.

\section{External affairs plan}

The closure of a mine can be complex and sensitive, involving public meetings, negotiations and media announcements. As in the social/community plan, this plan defines the strategy for the organisation's public relations function to manage external affairs, so that all closure personnel understand what information can be released and by whom.

While the requirements for specific community and public relations inputs appear logical and automatic, it is important that the planning team engage with these functions early in the closure planning process to fully understand and appreciate these aspects of the process which are critical to achieving relinquishment.

\section{Review, improvement, change management}

Prior to closure execution, closure plans need to be reviewed and updated on a fairly regular basis so that the information used in closure provisioning or decision making is up to date. It is therefore necessary to set out an anticipated timeline for the evolution of the detailed execution and post-closure plans so that these actions can be incorporated into the life of mine plan, shorter term plans, e.g. five year plan, and operating budgets.

The internal and external review and approval processes need to be clearly defined and scheduled. If possible, a joint regulatory review group should be established that includes both federal and state/provincial regulators. This will facilitate and simplify the review process and keep the approval process on schedule by ensuring that all information is shared across all applicable regulatory agencies and stakeholders.

As the closure management process can extend over many years, it is crucial that all data, information, correspondence and any changes are clearly and fully documented, including documenting the impacts that changes have on the closure scope, methodology, schedule, risk profile and cost, and how these have been managed.

It is recommended that a searchable, electronic reference library/database is established early in the project, in which all technical references, environmental assessments, design reports, as-built reports and drawings are referenced. 


\section{Acknowledgements}

Evelyn Bingham was responsible for implementing and achieving group-wide compliance with the 2004 BHP Billiton closure standard. Evelyn has also provided excellent insight into closure issues and much appreciated assistance in developing and reviewing the key closure considerations.

From experiences at Elliot Lake, Debbie Berthelot provided useful insights into defining closure criteria, pointers on good planning and management techniques, and valuable lessons learned, all of which have been incorporated in this paper.

\section{References}

BHP Billiton (2004) BHP Billiton Closure Standard, 2004 (now obsolete internal document).

Department of Industry, Tourism and Resources (DoITR) (2006) Australian Government DoITR Leading Practice Sustainable Development Guideline on Mine Closure and Completion, October 2006.

International Council on Mining and Metals (ICMM) (2008) Planning for Integrated Mine Closure: Toolkit, 2008. 